\title{
Morphometric geometric study of wing shape in Culex quinquefasciatus Say (Diptera: Culicidae) from Tamil Nadu, India
}

\author{
K. Manimegalai ${ }^{1}$, M. Arunachalam ${ }^{2} \&$ R. Udayakumari ${ }^{3}$ \\ ${ }^{1,3}$ Avinashilingam University for Women, Coimbatore, Tamil Nadu 641043, India \\ ${ }^{2}$ Sri Paramakalyani Centre for Environmental Sciences, Manonmaniam Sundaranar University, Alwarkurichi, Tamil Nadu 627412, India \\ Email: ${ }^{1}$ manijeysu@gmail.com; ${ }^{2}$ arunacm @ gmail.com (corresponding author)
}

Date of online publication 26 May 2009 ISSN 0974-7907 (online) | 0974-7893 (print)

\section{Editor: R. Ramanibai}

\section{Manuscript details: \\ Ms \# 01982 \\ Received 19 April 2008 \\ Final received 28 February 2009 \\ Finally accepted 29 April 2009}

Citation: Manimegalai, K., M. Arunachalam \& R. Udayakumari (2009). Morphometric geometric study of wing shape in Culex quinquefasciatus Say (Diptera: Culicidae) from Tamil Nadu, India. Journal of Threatened Taxa 1(5): 263-268.

Copyright: (c) K. Manimegalai, M. Arunachalam \& R. Udayakumari 2009. Creative Commons Attribution 3.0 Unported License. JoTT allows unrestricted use of this article in any medium for non-profit purposes, reproduction and distribution by providing adequate credit to the authors and the source of publication.

Author Details: K. Manimegalal, is a Reader and the fields of specialization are: control of vector species of mosquitoes using plant extracts, mosquito taxonomy using wing shape analysis.

M. Arunachalam, is a Professor and his fields of specialization are: stream / river / wetlands ecology, fish ecology, fish habitat mapping using GIS, fish taxonomy and phylogenetics, morphometric geometry of shape analysis of the wings of aquatic insects and mosquitoes and shape analysis of cypriniform fishes. R. UDAYAKUMARI was a M.Phil., Scholar

Acknowledgements: We acknowledge the help from Mr. M. Murugan, Sri Paramakalyani Centre for Environmental Sciences of Manonmaniam Sundaranar University. We also acknowledge the help from Prof. James R. Rohlf for his reply to our questions while using his programmes.
Abstract: The morphometric geometric study was carried out in 10 males and 10 females of Culex quinquefasciatus. There are 23 landmarks corresponding to points at which wing veins either branch or intersect the margin of the wing. Relative warp analysis has been proved to be very efficient in distinguishing the variation of shape in male and female wings. The multivariate analysis of co-variance (MANCOVA) showed a clear separation of the male and female wings.

Keywords: Culex quinquefasciatus, Geometric morphometrics, Landmarks, MANCOVA, Relative warp

\section{INTRODUCTION}

Landmark based geometric morphometrics have been increasingly used to explore systematic, developmental, ecological or pathological differences among individuals or populations, The approach of morphological landmarks is more appropriate as it represents advantages of additional information and the mode of variation is well understood (Bookstein 1989). Study of wing morphology in Lepidoptera and other insects using the geometric approach of shape variation provides an insight to taxonomy (Rohlf 1996). The genus Culex is a principal vector species in the peninsular India and the available keys are inadequate to study the morphological similarity of Culex species in this region and the present study is a pioneering attempt to distinguish the mosquito species based on wing morphometry. This paper forms a part of a major work on various aspects of different species of mosquitoes by the senior author.

\section{Materials and Methods}

Adult mosquitoes were collected from three localities in the Coimbatore city (the third largest city, $10^{\circ} 50^{\prime}-11^{\circ} \mathrm{O} 2^{\prime} \mathrm{N} \& 76^{\circ} 56^{\prime}-77^{\circ} \mathrm{O} 1^{\prime} \mathrm{E}$ in the Indian state of Tamil Nadu with an area of $1287 \mathrm{~km}^{2}$ with a population of $9,40,000$ and the elevation is $442 \mathrm{~m}$ above mean sea level). Wings were removed and photographed with consistent magnification and the digital images were used for the study. Landmarks (23) of the wings of 10 males and females of Culex quinquefasciatus were used for the analysis following the method of Rohlf \& Slice (1990). The landmarks are the intersections of wing veins with the wing margin, intersections of cross veins with major veins, vein branch points, the apparent anterior attachment of the wing to the body, and the notch between the posterior wing margin and the alula (Figs. 1-2). We digitized the photographs with the programme TPSDIG (Rohlf 1996a) and from the landmark coordinates we computed the consensus configuration using the programme TPSRELW (Rohlf 1997) which implements the algorithms described by Rohlf \& Slice (1990) and Bookstein (1996).

For each wing the uniform component of shape variation (affine) is estimated and the first uniform component accounts for the stretching along the x-axis of the configuration whereas the $2^{\text {nd }}$ uniform component indicates dilations or compressions along the y-axis. The uniform components were estimated by the Linearized Procrustes method of Bookstein (1996b). All wings were scaled to unit centroid size before alignment by the GLS super imposition method.

Thin-plate spline of relative warp analysis was carried out using the coordinates of all aligned wings (Bookstein 1991; Rohlf 1993) and this will give the display and direction of shape differences among the two species (herein the male and female wings of Culex quinquefasciatus). The Thin-plate splines technique (Bookstein 1989) has the function of interpolation to the landmark coordinates of each wing against the reference configuration in order to make the landmarks homologous. The bending energy matrix 

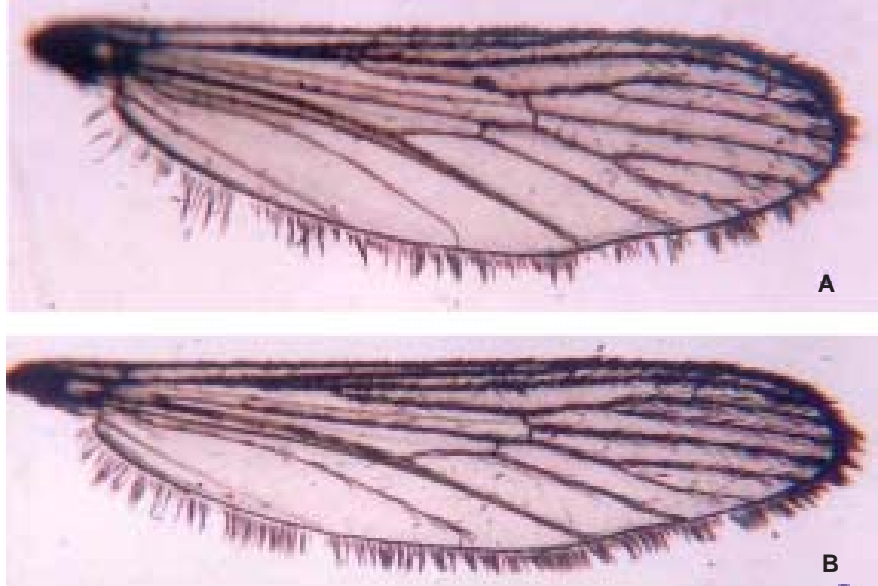

Figure 1. Culex quinquefasciatus A) Female, B) Male

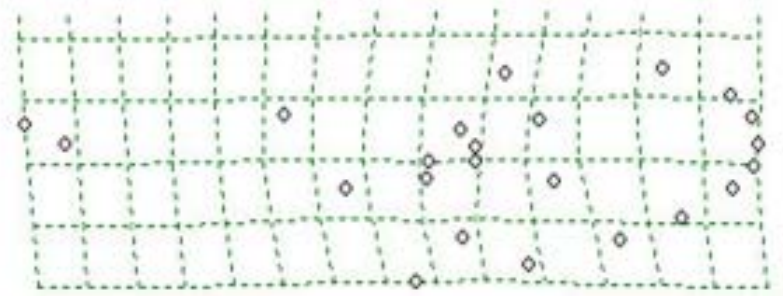

Parial warp 1

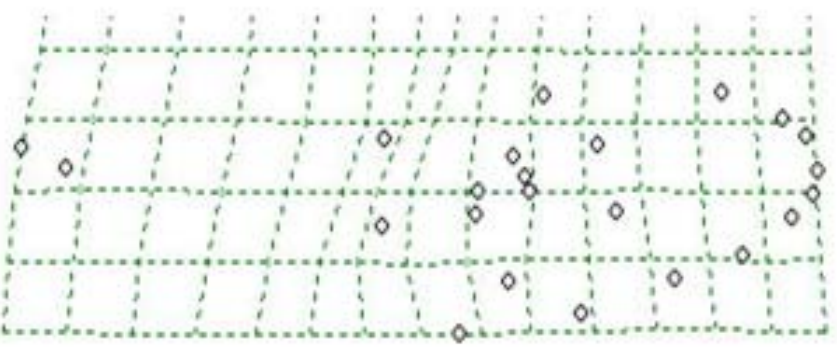

Parial warp 2

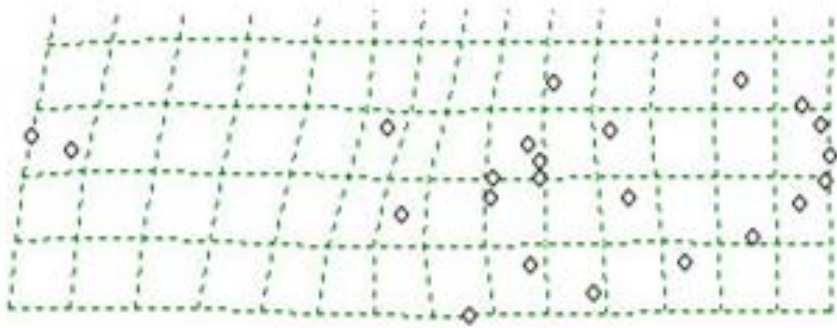

Parial warp 3

Figure 3. Plots of last three principal warps as displacement deformation equally for $x$ and $y$ coordinates (Alpha $=1$ )

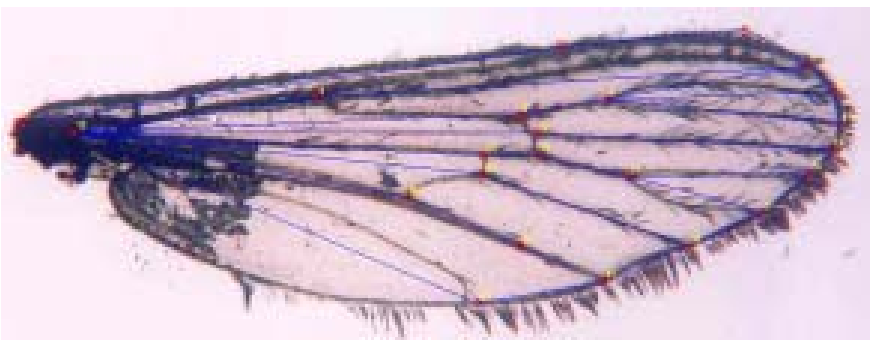

Figure 2. Plots of Culex quinquefasciatus used in the present study superimposed on the reference configuration using affine generalized resistant fit analysis. The reference configuration is shown with lines.

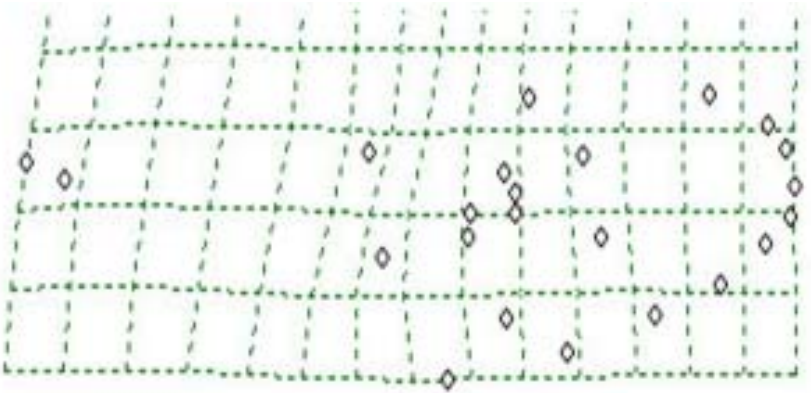

Relative Warp 1

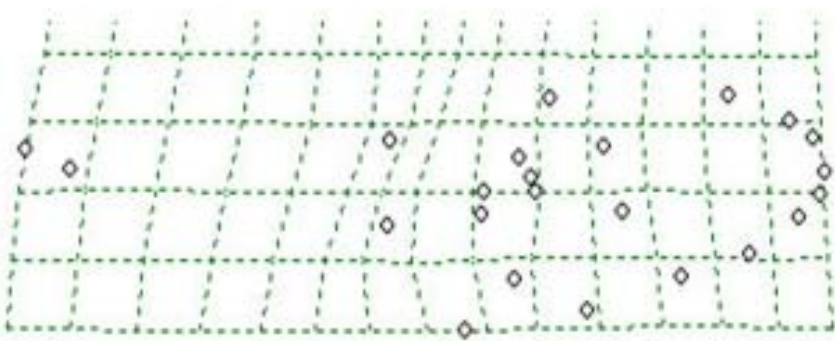

Relative Warp 2

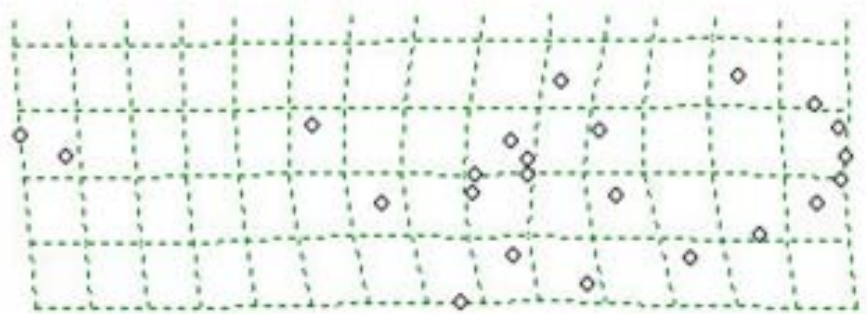

Relative Warp 3

Figure 4. Plots of the relative warp loadings (Alpha $=1)$ and the grid of the wings superimposed in the reference configuration based on affine generalised resistant fit (Alpha = 1) 


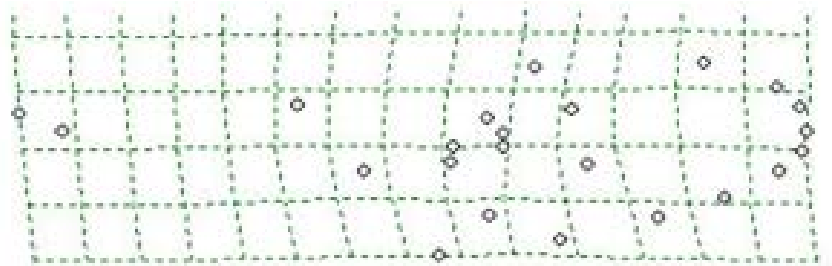

Relative Warp $1+$ deformation

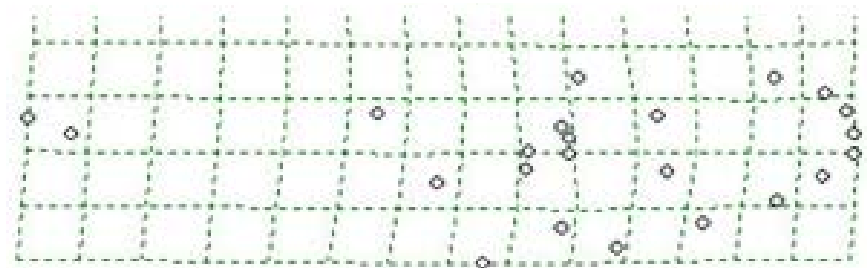

Relative Warp 1 - deformation

Figure 5. Plots of first two relative warps (based on Alpha $=1$ ) shown both as Thin - plate spline for positive and negative displacements along the first relative warp axes showing the net arrangement of land marks. (Alpha $=1)$

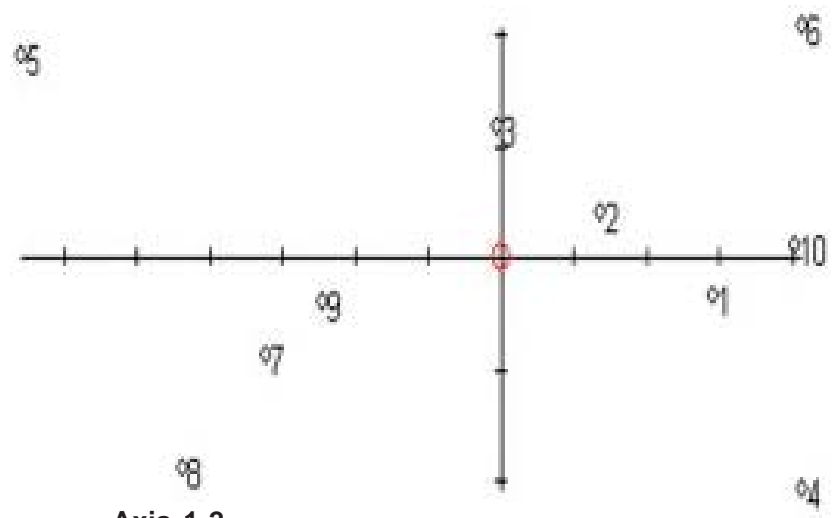

Axis 1-2

\section{$\phi$}
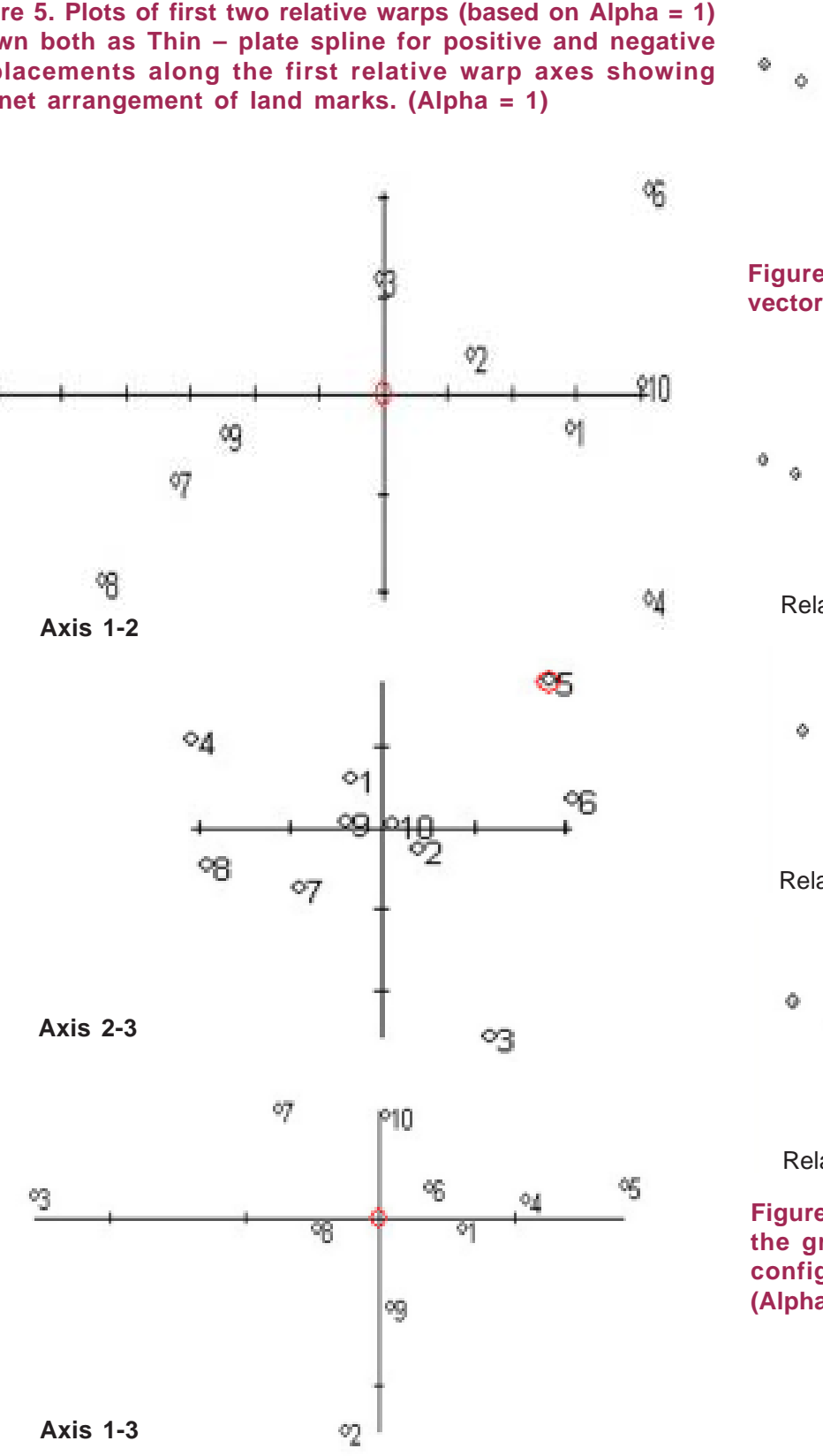

Figure 6. Ordination by the non-affine shape 1st, 2nd and 3rd components for Culex quinquefasciatus male and females (Alpha = 1)

Journal of Threatened Taxa | www.threatenedtaxa.org | May 2009 | 1(5): 263-268

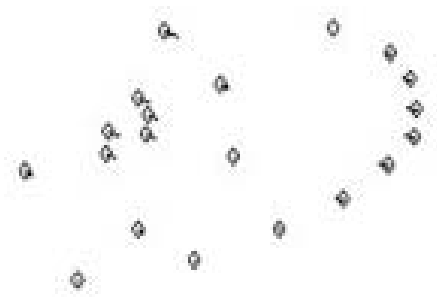

Parial warp 1

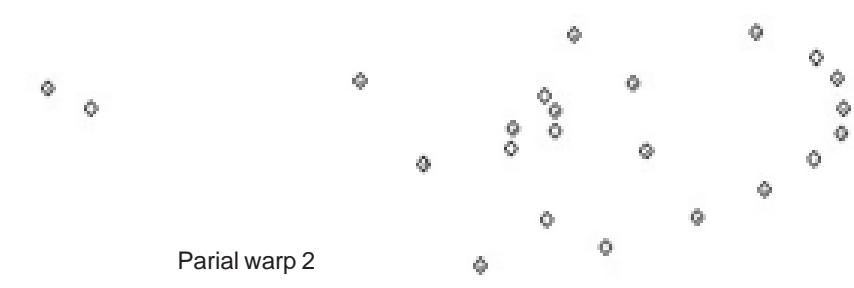

Parial warp 3

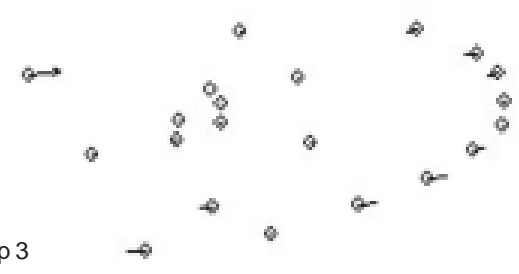

Figure 7. Plots of last three principal warps as displacement vector equally for $x$ and $y$ coordinates (Alpha $=0$ )

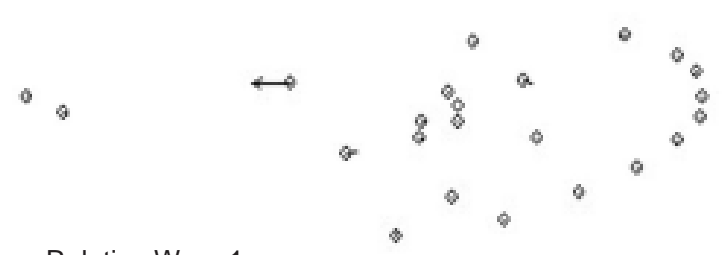

Relative Warp 1

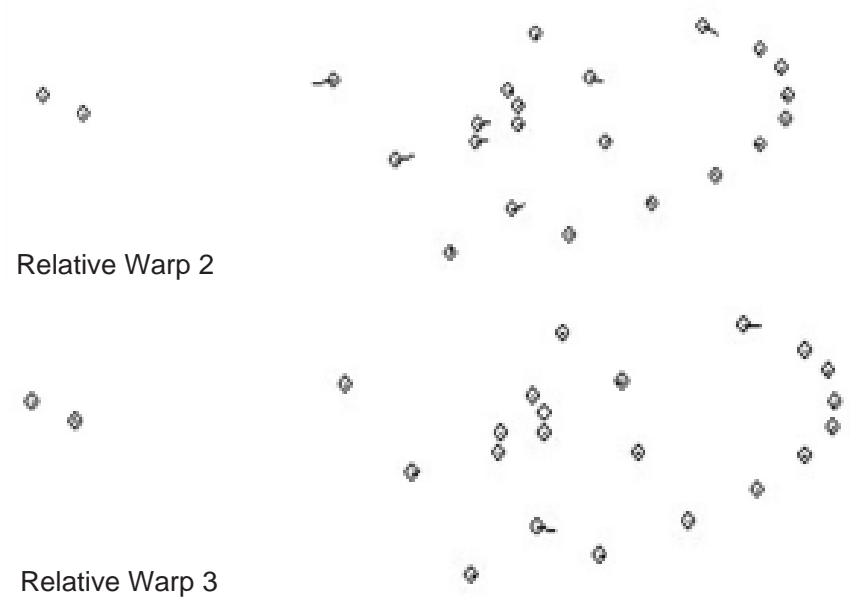

Figure 8 Plots of the relative warp loadings (Alpha $=0$ ) and the grid of the wings superimposed in the reference configuration based on affine generalised resistant fit $($ Alpha $=0)$ 


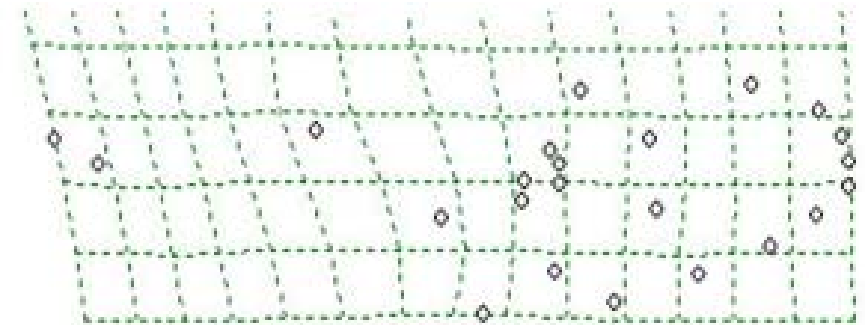

Relative warp $1+$ deformation

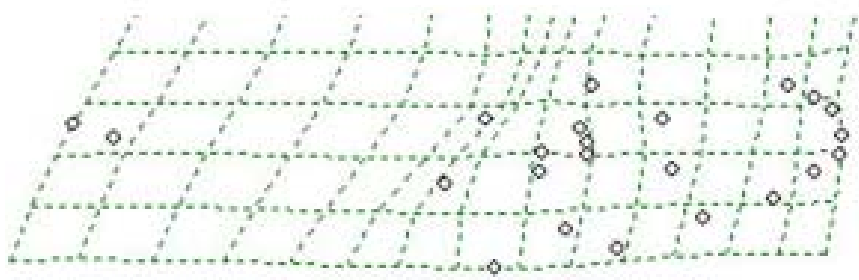

Relative warp 1 - deformation

Figure 9. Plots of first two relative warps (based on Alpha = o) shown both as Thin - plate spline for positive and negative displacements along the first relative warp axes showing the net arrangement of land marks. (Alpha $=0)$
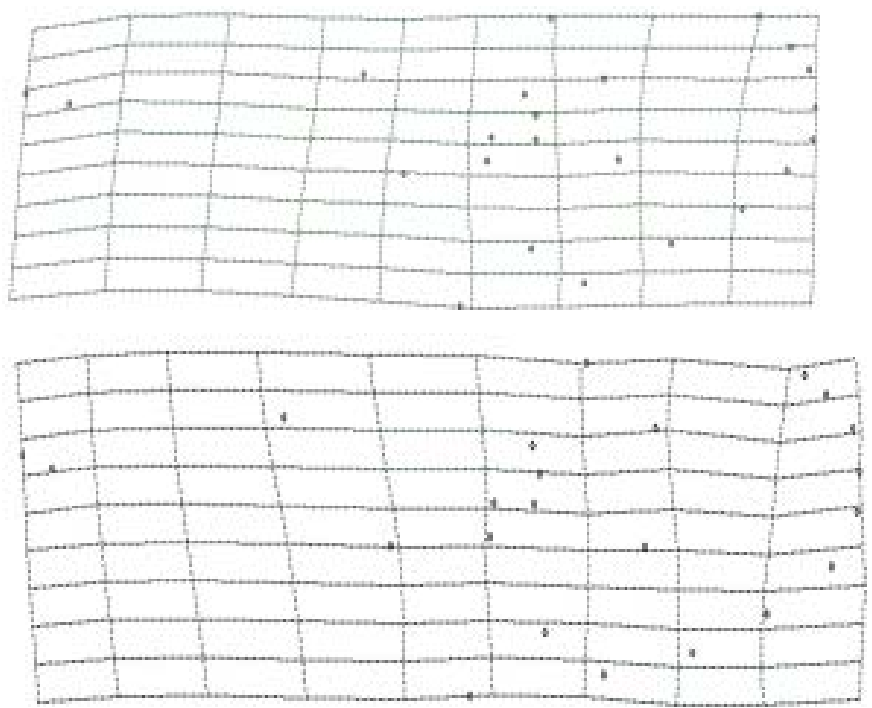

Figure 11. The non - affine deformation for the comparison between Cluex quinquefasciatus male and female

resulting from the thin-plate spline function fitted to the reference configuration will be modified as orthogonal axes which is called the principal warp which describe shape distortions of the reference configuration. The projection of the super imposed wings onto the principal warps produces the partial warp scores. These scores describe the deviations from the reference configuration. The relative warps are the principal components of the variation among wings (Bookstein 1989 \& 1991; Rohlf 1993). The coverage configuration of landmarks are used as the reference which is aligned to its principal axis. The relative warps are computed with the scaling option $\alpha=0$, which weights all landmarks equally. Otherwise all of the principal warps have the same weight and is considered to be more appropriate for systematic studies (Rohlf 1993; Rohlf et al. 1996). Weight matrix $\alpha=1$ corresponds to the relative warp analysis which shows variation among wings in the principal warps with larger bending

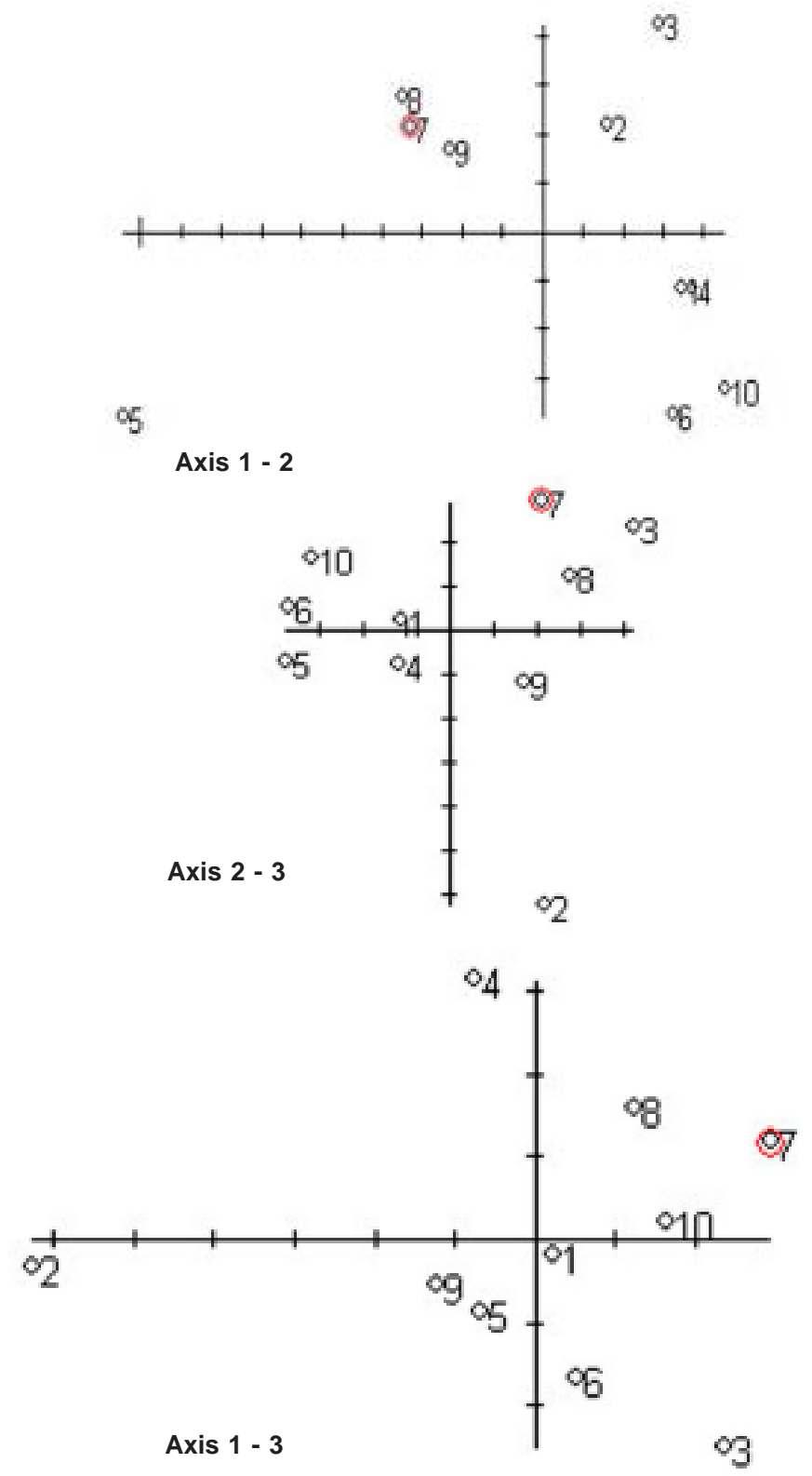

Figure 10. Ordination by the non-affine shape 1st, 2nd and 3rd components for Culex quinquefasciatus male and females $($ Alpha $=0)$.

energies.

Deformation grids using Thin-plate splines were used to graphically represent the patterns of shape variation among the landmarks. We analyzed the warp matrix with multivariate analysis of variance (MANCOVA).

\section{Results}

The positions of the landmark in the reference configuration are also indicated by the origin of vectors. The eigenvalue of the relative warp showed as 0.08482 and this is the square root of the variance per unit bending energy. The relative warp loadings showed values greater than zero (0.224, $0.145,0.125,0.1 .2,0.77,0.075,0.023,0.022$ and 0.041$)$ with the respective landmarks of $10,9,8,6,11,4,18,22$ and 14 (Figs. 3-5). 


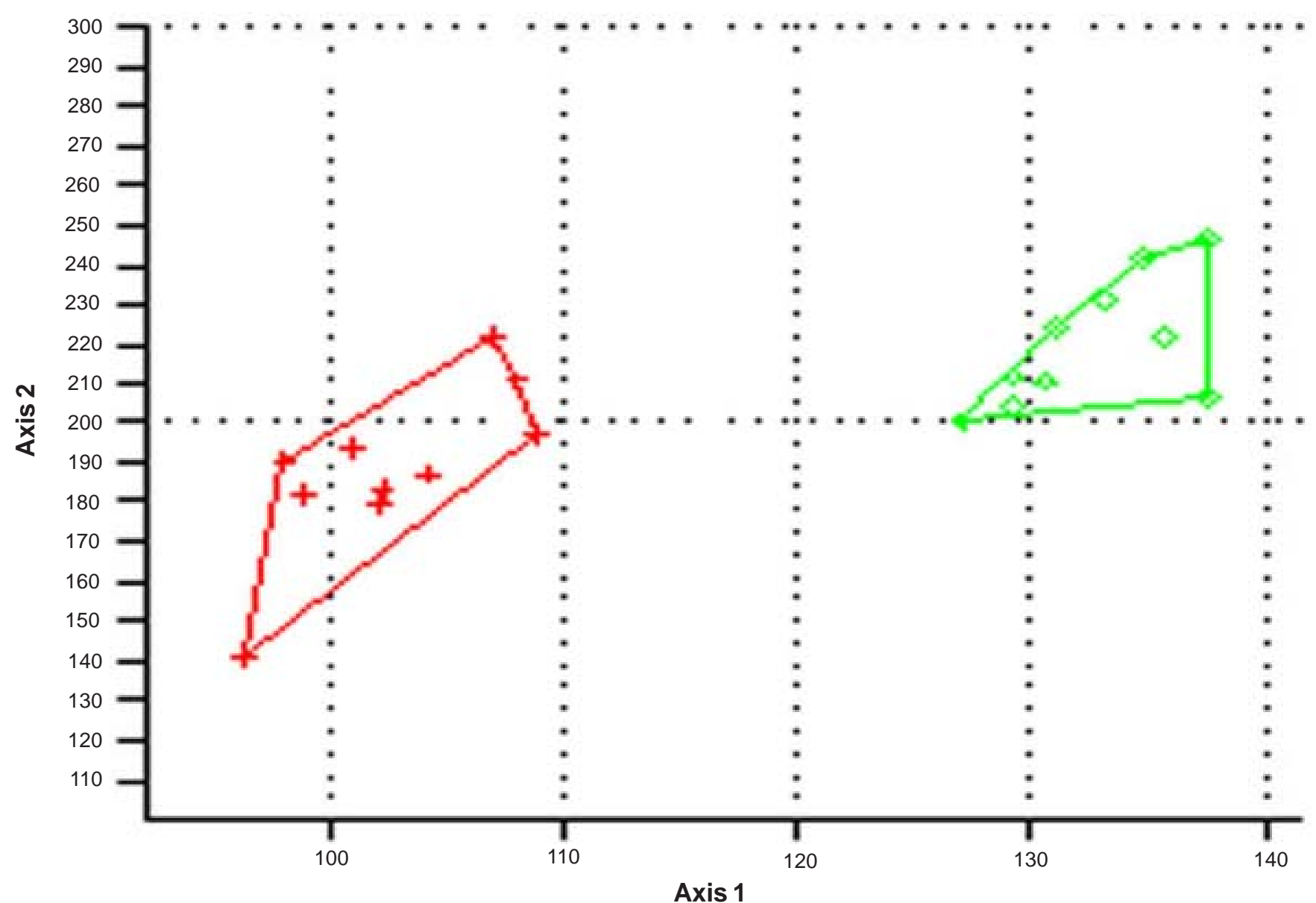

Figure 12. Polygons formed on the projection of male and female wings on to the MANCOVA variables 1 and 2.

The ordination of the non-affine shape of the first, second and third components of the Culex quinquefasciatus males and females are sharing the projections of partial warps towards the principal warps (Figs. 6-7). Most of the variations between male and female are along the axis 1-2.

The first relative warp has more variations than the second because in axis one it is on the left side. In the second axis it is on the right side and in axis 3 showing extreme right and left. It is obvious (Figs. -11 ) that the difference is between the base and tip of the wings. The affine components of males show variation of $93 \%$ and the females show $56 \%$ variation. The Thin-plate spline analysis shows that males show variation in the middle and distal ends while the female shows variation in the distal end of the wing.

It is also clear that the shape of the wings of male and female of Culex quinquefasciatus shows clear distinction based on landmark data with MANCOVA (Fig.12). Two polygons are so distinct illustrating the clear difference between the wing shapes of males and females.

\section{Discussion}

The multivariate analysis of co-variance (MANCOVA) showed a clear separation of male and female mosquitoes of Culex quinquefasciatus based on wing shape. There is no overlap of any landmark data between the male and the female and the data of selected pairs of point are the distance measurements used as multidimensional space (Goodall 1991).
The use of a parameter in this study following Rohlf (1993) is to identify appreciable changes in the value less than zero which will result in more weight to the small-scale features. In taxonomic studies $\alpha=0$ gives an equal weighting in the analysis and in growth study to record the allometry where differences are more and hence $\alpha=1$ is also used. As the present study reveals variations between male and female of Culex quinquefasciatus the study of using $\alpha=1$ is tried to find out any interesting patterns at different scales.

The bending energy concept used in the study is the quantity of the physical properties of the thin metal sheets and used as Thin-plate spline and the functions used in organisms do not imply biological significance to the parameter. The use of spline (Rohlf 1993) does not imply that biological tissues behave like metal sheets but it is simply a convenient function that is able to express the differences in two configurations of landmarks as a continuous deformation. This Thin-plate spline is the assessment of shape deformations such as bio-orthogonal grids (Bookstein et al. 1985).

The Procrustes plots in the relative levels of variation at different levels are difficult to find out the displacement of landmarks. Hence, the method of relative warps has been used to identify the co-variation very effectively. From this study one can easily identify the movement of landmark either towards the base of the wings or towards the tip of the wings. 


\section{References}

Bookstein, F.L., B. Chernoff, R.L. Elder, J.M. Humphries, G.R. Smith \& R.E.Strauss (1985). Morphometrics in Evolutionary Biology. Special Publication 15, Academy of Natural Sciences of Philadelphia, Philadelphia, 277pp.

Bookstein, F.L. (1989). Principal Warps: Thin - plate splines and the decomposition of deformations. IEEE Transactions on Pattern Analysis and Machine Intelligence 11: 567-585.

Bookstein, F.L. (1991). Morphometric Tools for Landmark Data. Geometry and Biology. Cambridge University Press, 435pp.

Bookstein, F.L. (1996a). A standard formula for the uniform shape component in landmark data, pp. 153-168. In: Marcus, L.F., M. Corti, A. Loy, G. Naylor \& De Slice (eds.), Advances in Morphometrics. Plenum Publishing Corps. New York.

Goodall, C.R. (1991). Procrustes methods in the statistical analysis of shape, Journal of Royal Statistical Society. Series. B 53: 285-339

Rohlf, F. J.\& D. Slice (1990). Extensions of the procrustes method for the optimal super impostion of landmarks. Systematic Zoology 39: $40-59$.

Rohlf, F.J. (1993). Relative warp analysis and an example of its applications to mosquito wings, pp. 131-159. In: Marcus, L.F., E.
Bello, A. Garcia Valdecasas (eds.), Contributions to Morphometrics. Monografia del Museo Nacional de Ciencias Naturales 8.Madrid.131159.

Rohlf, F.J., A. Loy \& M. Corti (1996). Morphometric analysis of old world Talpidae (Mammalia, Insectivora) using partial warp scores.

Systematic Biology 45: 344- 362.

Rohlf. F.J. (1996a). TPSDIG. Program of digitizing images for analaysis by Thin plate splines (Windows) Ver. 1.08, Department of Ecology and Evolution, State University of New York, Stony Brook.

Rohlf, F.J. (1996b). Morphometric spaces, shape components and the effects of linear transformations, pp. 117-129. In: L.F. Marcus, M. Corti, A. Loy, G. Naylor, D.E. Slice (eds.) Advances in Morphometrics. NATO Advance studies Institute on Morphometrics in II Ciocco, Italy. Plenum Publishing Corp, New York.

Rohlf, F.J. (1997). TPSRELW. Program for analyzing landmark data with Thin-plate spline using relative warps (Windows) Ver.1.14, Department of Ecology and Evolution, State University of New York, Stony Brook. 